\title{
Chitosan-modifizierte Sensoren für die elektrochemische Bestimmung von Sulfat- und Eisenionen
}

\author{
Anastasiya Svirepa ${ }^{1,2}$, Johannes Schwarz ${ }^{1}$, Kathrin Trommer ${ }^{1}$, Simona Schwarz ${ }^{3}$, Michael Mertig', \\ ${ }^{1}$ Kurt-Schwabe-Institut für Mess- und Sensortechnik Meinsberg e.V., Waldheim, Deutschland \\ ${ }^{2}$ Physikalische Chemie, Mess- und Sensortechnik, TU Dresden, Dresden, Deutschland \\ ${ }^{3}$ Leibniz-Institut für Polymerforschung Dresden e.V., Dresden, Deutschland \\ Kontakt: anastasiya.svirepa@ksi-meinsberg.de
}

\section{Einleitung}

Die langjährige, großflächige Braunkohlegewinnung in den Lausitzer Tagebauen des Mitteldeutschen Reviers führte durch Absenkung des Grundwasserspiegels zur erhöhten Verwitterung von Pyrit und Markasit in tieferliegenden geologischen Schichten. Infolge stieg die Konzentration von Sulfat- und Eisenionen in nahegelegenen Gewässern erheblich an. Durch die Oxidation von zweiwertigem Eisen wurde Eisenhydroxid in Form von Braunschlamm ausgefällt. Dadurch erhielt die Spree eine starke bräunliche Färbung, was als „Verockerung“ bezeichnet wird [1].

$$
\begin{aligned}
& \mathrm{FeS}_{2}+3,5 \mathrm{O}_{2}+\mathrm{H}_{2} \mathrm{O} \rightarrow \mathrm{Fe}^{2+}+2 \mathrm{SO}_{4}^{2-}+2 \mathrm{H}^{+}, \\
& 4 \mathrm{Fe}^{2+}+\mathrm{O}_{2}+10 \mathrm{H}_{2} \mathrm{O} \rightarrow 4 \mathrm{Fe}(\mathrm{OH})_{3} \downarrow+8 \mathrm{H}^{+}
\end{aligned}
$$

Als Ergebnis des Eingriffs in den Grundwasserhaushalt besteht dieses Umweltproblem sowohl in Brandenburg als auch in Sachsen, wobei die Spree besonders betroffen ist. Die Konzentration von Sulfationen im Gewässer beträgt $\leq 2000 \mathrm{mg} / \mathrm{l}$ und der Eisengehalt kann bei bis zu $80 \mathrm{mg} / \mathrm{l} \mathrm{lie-}$ gen. Eine hohe Konzentration der beiden Schadstoffe führt zur Belastung von Naturlebensräumen, Schädigung von Bauwerken wie Brücken und kann auch die Qualität des Trinkwassers beeinträchtigen. Eine schnelle Vor-Ort-Analyse des Sulfat- und Eisengehaltes ist daher von großer Bedeutung.

Chitosan ist ein nicht giftiges, biokompatibles und biologisch abbaubares Polymer, welches aus den Schalen von Krebstieren durch Deacetylierung des Chitins, d. h. durch Abspaltung der $\mathrm{N}$-Acetylgruppe, gewonnen wird. Der Deacetylierungsgrad (engl. degree of deacetylation (DD), in \%) wird als Reinheitsgrad des Chitosans bezeichnet [2].

Das Chitosanmolekül enthält eine große Anzahl von freien Aminogruppen, welche im sauren $\mathrm{pH}-$ Bereich protonieren:

$$
\text { Chit- } \mathrm{NH}_{2}+\mathrm{H}_{2} \mathrm{O} \rightarrow \text { Chit- } \mathrm{NH}_{3}{ }^{+}+\mathrm{OH}^{-} \text {. }
$$

Positiv geladene Aminogruppen verleihen dem Polymer besondere Eigenschaften. Daraus ergibt sich die Fähigkeit von Chitosan, ein gutes Adsorptions- und Flockungsmittel für die Behandlung und Reinigung von kontaminierten Gewässern zu sein. Als ein guter lonenaustauscher adsorbiert Chitosan Ionen verschiedener Metalle. Durch die Bildung von Wasserstoffbrücken ist das Biopolymer in der Lage, organische Substanzen zu binden. Diese besonderen Eigenschaften eröffnen damit ein breites Einsatzspektrum von
Chitosan. Eine der innovativsten Anwendungen stellt die elektrochemische Sensorik dar.

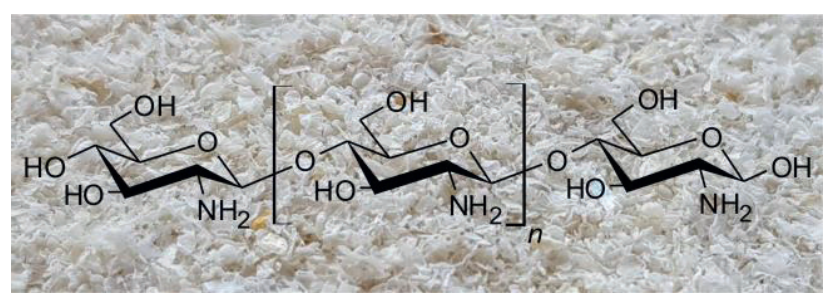

Abb. 1: Chitosan-Flocken und Strukturformel.

Biopolymere wie Chitosan stellen eine vielversprechende Alternative zu synthetischen Analyt-komplexierenden Verbindungen bei der Entwicklung von elektrochemischen Sensoren dar. Das Ziel der Arbeit ist die Entwicklung spezifischer, elektrochemischer, Biopolymer-basierter All-Solid-State-Sensoren für das Monitoring von Sulfat- und Eisenionen in der Spree. Speziell soll hier demonstriert werden, dass eine Chitosan-modifizierte Arbeitselektrode mit einer PVC-Membran sowohl für die potentiometrischen Messungen als auch für die voltammetrischen Bestimmungen eingesetzt werden kann.

\section{Methoden und Materialien}

Präparation von Graphitpaste-basierten Elektroden

Die Graphitpaste wurde durch manuelles Mischen von $51 \%$ (w/t) Graphitpulver, 43 \% (w/t) Harz und $6 \%$ (w/t) Härter hergestellt. Die Mischung wurde in Elektrodenkörper aus PVC mit darin befindlichem Pt-Draht als Ableitung gepresst. Nach dem Aushärten wurde die Elektrodenoberfläche geschliffen. Die resultierende Oberfläche der Arbeitselektrode beträgt $0,126 \mathrm{~cm}^{2}$ (ø $4 \mathrm{~mm}$ ).

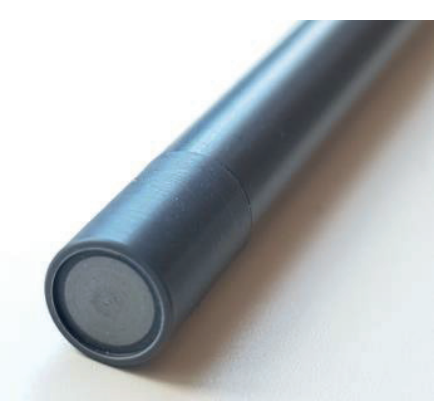

Abb. 2: Graphitpaste-basierte Elektrode. 
Beschichtung der Elektrodenoberfläche mit Polymer.

Die leitfähigen Polypyrrolfilme (PPy) wurden als Mediatorschicht durch In-situ-Elektropolymerisation des Monomers Pyrrol (Py) mittels Cyclovoltammetrie (CV) an den Oberflächen der Graphitpaste-basierten Arbeitselektroden hergestellt. Als Referenzelektrode und Gegenelektrode wurden entsprechend eine $\mathrm{Ag} / \mathrm{AgCl}_{\text {sat.KCl}}$-Elektrode und ein Platindraht verwendet. Die elektrochemische Abscheidung erfolgte aus einer 0,1 mol/l Pyrrol-Lösung in 0,1 mol/ $\mathrm{KNO}_{3}$. Die Ströme wurden im Potentialbereich zwischen $0,2 \mathrm{~V}$ und $0,7 \mathrm{~V}$ mit einer Abtastrate von 0,01 V/s und einem Potentialschritt von $5 \mathrm{mV}$ aufgenommen. Insgesamt wurden 100 Zyklen durchgeführt.

\section{Herstellung einer ionensensitiven Membran}

Die 2\%tige-Chitosanlösung wurde durch Lösen von $0,06 \mathrm{~g}$ Chitosan in $3 \mathrm{ml}$ Essigsäure $(2 \mathrm{~mol} / \mathrm{l})$ unter Rühren für $18 \mathrm{~h}$ hergestellt. Polyvinylchlorid (PVC) und Weichmacher 2-Nitrophenyloctylether (2-NPOE) wurden zuerst in Tetrahydrofuran (THF) gelöst. Anschließend wurde die Chitosanlösung zugegeben. Die Membran enthält Anteile von 48,38 \% (w/t) PVC, 48,38 \% (w/t) 2-NPOE und 3,24 \% (w/t) Chitosan. Auf die mit Polypyrrol beschichtete Oberfläche der Arbeitselektrode wurden $40 \mu \mathrm{l}$ der Mischung durch Drop-casting aufgetragen.

\section{Materialien und Geräte}

Für die Herstellung der Paste wurden Graphitpulver (Fluka), Epoxidharz Epilox ${ }^{\circledR}$ T19-32/1000 (Leuna-Harze $\mathrm{GmbH}$ ) und Härter DPTA (Epoxidharze Andreas Weigel) verwendet. Chitosan in Form von Pulver mit einem DD von 90\% (Produktname Ch90/30/A1) wurde von der Firma BioLog Heppe ${ }^{\circledR} \mathrm{GmbH}$ bereitgestellt. Pyrrol, hochmolekulares PVC, THF und 2-NPOE wurden von Sigma Aldrich bezogen. Die Salze für die Herstellung von Messlösungen wurden von Carl Roth erworben.

Cyclovoltammetrie und Differential-Pulse-Voltammetrie (DPV) wurden mit dem PalmSens Multi EmStat 2 und der PSTrace-Software von PalmSens durchgeführt. Die potentiometrischen Messungen erfolgten mit dem Labormesssystem LM 2000 und der Software LM remote von Xylem Analytics Germany Sales $\mathrm{GmbH}$ \& Co. KG Sensortechnik Meinsberg. Die REM-Aufnahmen erfolgten mit Helios NanoLab ${ }^{\mathrm{TM}} 660$.

\section{Ergebnisse und Diskussion}

\section{Elektropolymerisation von Pyrrol}

Leitfähiges Polypyrrol stellt einen lon-to-electron-Transducer zwischen der ionisch leitenden PVC-Membran und dem elektrisch leitenden graphithaltigen Substrat im chemischen Sensor dar [3, 4]. Abb. 3 zeigt die Oxidations-Reduktions-Kurven der Elektropolymerisation durch CV. Als hellblaue und dunkelblaue Messkurven sind der erste CV-Zyklus bzw. der 100. CV-Zyklus dargestellt. Die anderen Messkurven zeigen jedes zehnte Voltammogramm. Als Inset ist eine vereinfachte Oxidationsreaktion von Py zu PPy abgebildet:

$$
\mathrm{Py} \rightarrow \mathrm{PPy}+\mathrm{e}^{-}
$$

Der Mechanismus der Gesamtreaktion zur Bildung des Polymerfilmes ist kompliziert und umfasst mehrere Reaktionsschritte [5]. Der Anstieg der anodischen Stromdichte mit steigendem Potential ist mit der anodischen Oxidationsreaktion des Monomers verbunden. Die folgende Änderung der kathodischen Stromdichte entspricht der Bildung bzw. dem Wachstum des Polymerproduktes [6].

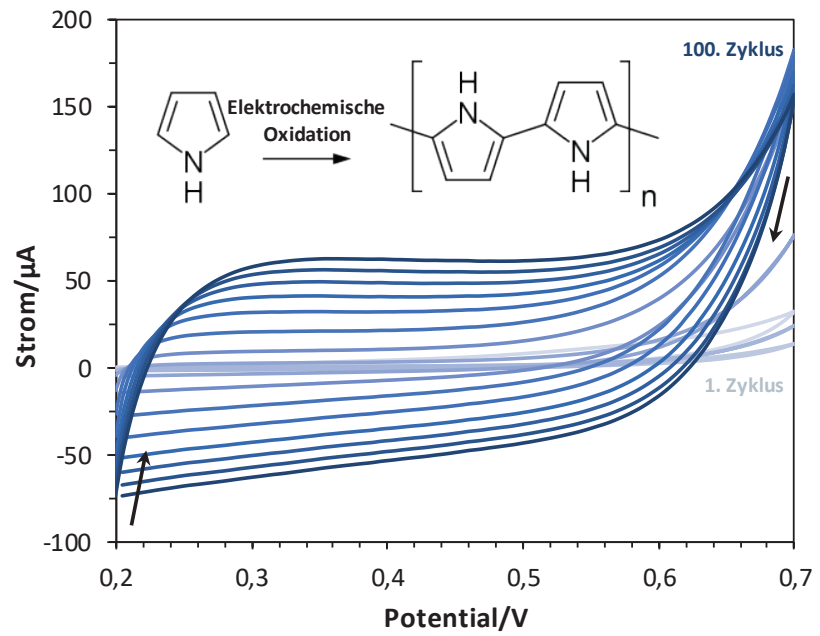

Abb. 3: Elektropolymerisation von leitfähigem Pyrrol mit Cyclovoltammetrie.

Die rasterelektronenmikroskopischen (REM) Aufnahmen einer Elektrodenoberfläche ohne PPy und einer PPy-beschichteten Oberfläche sind in der Abb. 4 zu sehen.
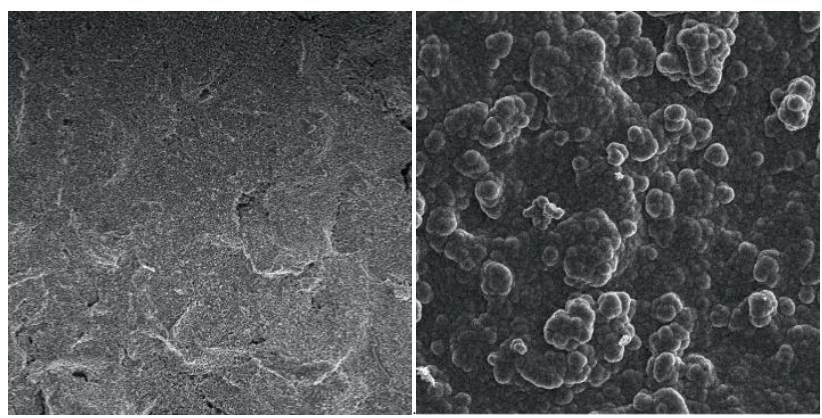

Abb. 4: REM-Aufnahmen der Elektrodenoberflächen ohne PPy-Film (links) und mit PPy-Film (rechts) bei 5.000-facher Vergrößerung.

Die Dicke des abgeschiedenen Polypyrrols ist für die Stabilität des Films von großer Bedeutung und soll $10 \mu \mathrm{m}$ nicht überschreiten [7]. Im anderen Fall löst sich der Polymerfilm von der Oberfläche ab. Zur Bestimmung der Filmdicke $h$ wurde das Faraday'sche Gesetz der Elektrolyse verwendet:

$$
h=\frac{Q M}{A z F}
$$

mit $Q$ - elektrische Ladung, $M$ - Molmasse Pyrrol, $A$ - Fläche der Elektrode, $z$ - Wert des lons und $F$ - Faraday-Konstante. Die resultierende Filmdicke liegt bei $3 \mu \mathrm{m}$. 


\section{Potentiometrische Bestimmung von Sulfationen}

Die potentiometrische Bestimmung von Sulfationen mit einer Chitosan-modifizierten Elektrode erfolgte in $\mathrm{Na}_{2} \mathrm{SO}_{4}-$ Standardlösungen unterschiedlicher Konzentration. Das potentiometrische Ansprechverhalten, gemessen gegen eine $\mathrm{Ag} / \mathrm{AgCl}$-Referenzelektrode, ist in der Abb. 5 zu sehen. Als Inset ist die resultierende Kalibrierkurve eingefügt. Es wurden reproduzierbare und stabile Potentialwerte erhalten. Im untersuchten Konzentrationsbereich wurde eine durchschnittliche Steilheit von 22,3 mV/Dekade berechnet. Das Bestimmtheitsmaß $R^{2}$ der linearen Regression beträgt 0,99 .

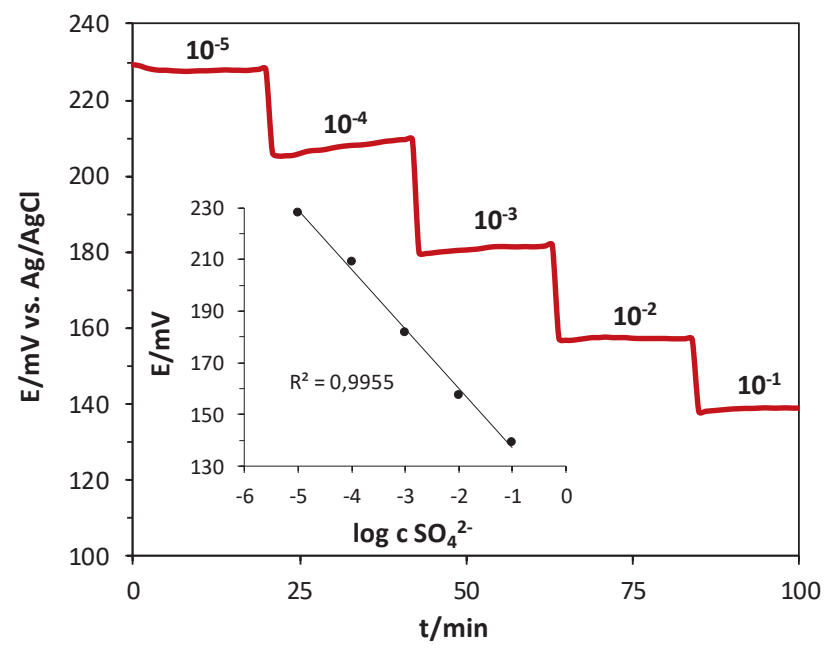

Abb. 5: Potentiometrisches Ansprechverhalten einer Chitosan-modifizierten Elektrode in $\mathrm{Na}_{2} \mathrm{SO}_{4}$-Standardlösungen $\left(10^{-5} \mathrm{~mol} / \mathrm{l}\right.$ bis $\left.10^{-1} \mathrm{~mol} / \mathrm{l}\right)$ und Kalibrierkurve (Inset).

\section{Voltammetrische Bestimmung von Eisenionen}

Eine Arbeitselektrode gleicher Modifikation wie im potentiometrischen Versuch wurde für die voltammetrische Detektion von Eisenionen eingesetzt (Abb. 6). Eine Referenzelektrode auf Harz-KCl-Basis und Pt-Draht wurden für die Messungen verwendet.

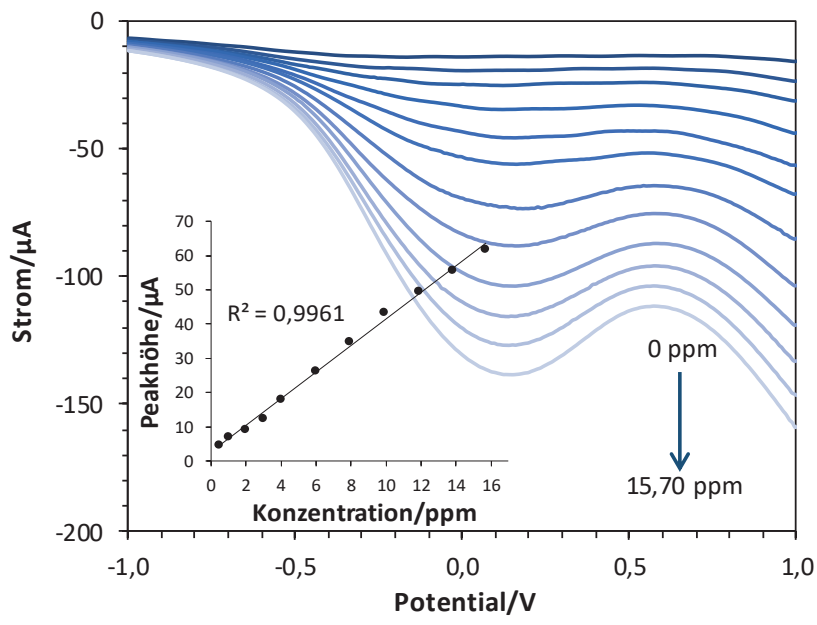

Abb. 6: $\mathrm{Fe}^{3+}$-Bestimmung an Chitosan-modifizierter Elektrode durch adsorptive Stripping-Voltammetrie mit Kalibrierkurve (Inset) im Konzentrationsbereich bis 15,7 ppm.
Die voltammetrischen Messungen erfolgten im DPV-Modus. Als Elektrolyt wurde $10^{-4} \mathrm{~mol} / \mathrm{l} \mathrm{KCl}$-Lösung mit $3 \times 0,001 \mathrm{~mol} / \mathrm{l}$ Ethylendiamintetraessigsäure (EDTA) verwendet. EDTA gehört zur Gruppe der Aminopolycarbonsäure-Liganden. Über sechs komplexbildende Gruppen, nämlich zwei Stickstoff- und vier Carboxylgruppen, bildet EDTA starke Komplexe mit den $\mathrm{Fe}^{3+}$-lonen [8].

Das Messprinzip der $\mathrm{Fe}^{3+}$-Bestimmung durch Adsorptions-Stripping-Voltammetrie ist in der Abb. 7 schematisch dargestellt. Die Methode beruht auf der Komplexierung von dreiwertigem Eisen mit Ethylendiamintetraessigsäure. Nach der Zugabe eines $\mathrm{Fe}^{3+}$-Aliquots zum EDTA-haltigen

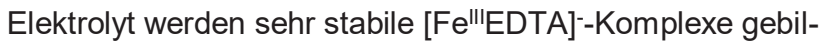
det. Diese negativ geladenen Komplexverbindungen reagieren mit positiv geladenen Aminogruppen von Chitosan und adsorbieren damit auf der Elektrodenoberfläche. Der Prozess verläuft sehr schnell und ohne elektrochemische Abscheidung durch Anlegen eines Potentials über eine bestimmte Zeit. Beim Stripping-Schritt wird das Potential in kathodische Richtung von $+1,0 \mathrm{~V}$ bis $-1,0 \mathrm{~V}$ gefahren. Während die [Fe $\left.{ }^{\prime \prime \prime} E D T A\right]^{-}-K o m p l e x e ~ z u ~[F e " l E D T A]^{2-}$ reduzieren, wird ein kathodischer Strompeak beim Potential von ca. $+0,1 \mathrm{~V}$ registriert. Mit dem Stromsignal der Peakhöhe wurde eine Kalibrierkurve erstellt. Das Bestimmtheitsmaß $\mathrm{R}^{2}$ der linearen Regression liegt bei 0,99.

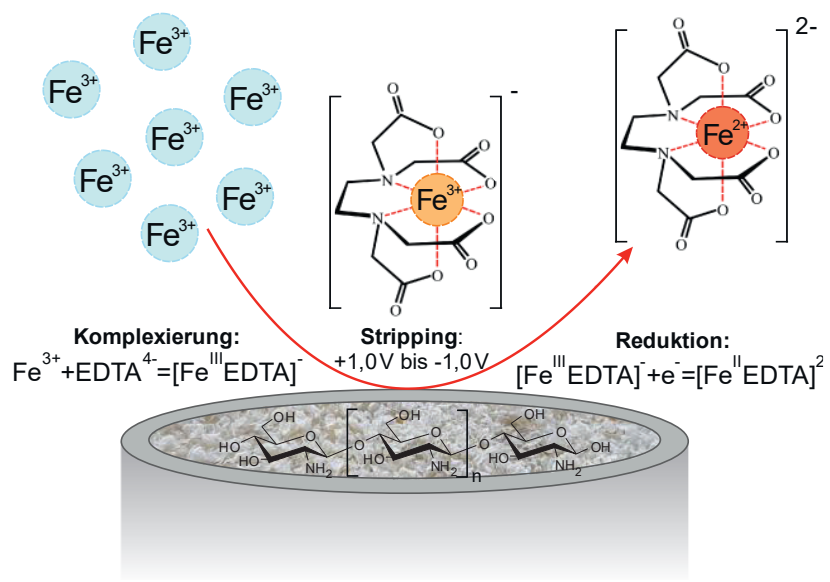

Abb. 7: Schematische Darstellung der $\mathrm{Fe}^{3+}$-Bestimmung an einer Chitosan-modifizierten Elektrode durch adsorptive Stripping-Voltammetrie.

\section{Zusammenfassung und Ausblick}

Das Biopolymer Chitosan wurde als umweltfreundliche Alternative zu synthetischen lonophoren bei der Herstellung von elektrochemischen Sensoren verwendet. Durch Immobilisierung von Chitosan in die PVC-Membran wurden potentiometrische Sulfat-sensitive Elektroden entwickelt und in Standardlösungen erprobt. Die Innovation besteht in der Möglichkeit, genau diese Elektrodenmodifikation ebenfalls für die voltammetrische Bestimmung anzuwenden. Die $\mathrm{Fe}^{3+}$-Bestimmung durch adsorptive Stripping-Voltammetrie mit einer Chitosan-modifizierten Elektrode war erfolgreich. 
Für einen zukünftigen Einsatz der Elektroden in Realproben von Spreewässern ergeben sich gute Anwendungsmöglichkeiten. Erstmalig wurden ionenselektive Elektroden der gezeigten Bauweise und Modifikation sowohl für die potentiometrische Detektion von Sulfationen als auch für die voltammetrische Bestimmung von Eisenionen verwendet.

\section{Literatur}

[1] Pyritverwitterung: Sächsisches Landesamt für Umwelt, Landwirtschaft und Geologie, https://www.umwelt.sachsen.de/umwelt/26953.htm, 22.03.2020

[2] SILVA, S. B., BATISTA, G. L., SANTIN, C. K.: Chitosan for Sensors and Electrochemical Applications. In: Chitin and chitosan: Properties and applications; Wiley, Hoboken, NJ, 2020, S. 461-476, ISBN 978-1-11945043-6

[3] CAMMANN, K., GALSTER, H.: Das Arbeiten mit ionenselektiven Elektroden. Berlin, Heidelberg, Springer Berlin Heidelberg, 1996

[4] MICHALSKA, A.: Optimizing the analytical performance and construction of ion-selective electrodes with conducting polymer-based ion-to-electron transducers. In: Analytical and bioanalytical chemistry 384 (2006), Nr. 2, S. 391-406

[5] DIAZ, A. F., CASTILLO, J. I., LOGAN, J. A., LEE, W.Y.: Electrochemistry of conducting polypyrrole films. In: Journal of Electroanalytical Chemistry and Interfacial Electrochemistry 129 (1981), Nr. 1-2, S. 115-132

[6] SABOURAUD, G., SADKI, S., BRODIE, N.: The mechanisms of pyrrole electropolymerization. In: Chemical Society Reviews 29 (2000), Nr. 5, S. 283-293

[7] DEJEU, J., TAOUIL, A. E., ROUGEOT, P., LAKARD, S., LALLEMAND, F., LAKARD, B.: Morphological and adhesive properties of polypyrrole films synthesized by sonoelectrochemical technique. In: Synthetic Metals 160 (2010), Nr. 23-24, S. 2540-2545

[8] BERGE, H., DRESCHER, A.: Indirekte inversvoltammetrische Bestimmung von Elementen unter Anwendung von Verdrängungsreaktionen. In: Fresenius' Zeitschrift für analytische Chemie 231 (1967), Nr. 1, S. 1117

\section{Danksagung}

Diese Arbeit wird in Kooperation mit dem Leibniz IPF Dresden im Projektvorhaben "Biopolymerbasierte SensorAktor-Systeme zur Detektion und Flockung von Schadstoffen in verockerten Oberflächenwässern" (Akronym: DeFloWasser, Projektnummer: 100377118) durchgeführt.

Die Autoren danken der Sächsischen Aufbaubank (SAB) für die Förderung des Projektvorhabens.

Das Kurt-Schwabe-Institut für Mess- und Sensortechnik Meinsberg e.V. wird mitfinanziert durch Steuermittel auf der Grundlage des vom Sächsischen Landtag beschlossenen Haushaltes. 